\title{
Die Sündenvergebung bei Irenäus.
}

Von Prof. Dr. Hugo Koch in Braunsberg.

In der letzten Zeit ist auf katholischer Seite von dem Dogmatiker G. Esser ${ }^{1}$ in Bonn und dem inzwischen heimgegangenen Kirchenhistoriker F.X.F unk $^{2}$ in Tübingen über Tertullians Stellung zur Buße und die Tragweite des Kallistschen Indulgenzediktes gestritten worden. Jener glaubt dem Afrikaner einen Gesinnungswechsel in der Bubfragre nachweisen zu können, insofern dieser in seiner späteren montanistischen Schrift De pudicitia als Neuerung bekämpfte, was er selbst in der Schrift De paenitentia aus seiner katholischen Zeit anerkannt habe - eine Bußse der Unzuchtssünder mit kirchlicher Rekonziliation. Diese habe es also in Karthago und Rom, wie übcrhaupt in den größeren Kirchen des Abendlandes schon damals gegeben, und das Edikt Kallists habe nicht eine neue Praxis eingeführt, sondern nur einer schon bestehenden, aber nicht allgemein anerkannten zum siegreichen Durchbruch verholfen. Dem gegenüber hält Funk in eingehender Auseinandersetzung an seiner früher schon begründeten und auch in seinen "Kirchengeschichtlichen Abhandlungen und Untersuchungen" I (1897) I55-181 vertretenen Auffassung fest, wonach Tertullian in De paenitentia zwar eine kirchliche Bulje mit Aussicht auf göttliche Verzeihung, aber keine kirchliche Rekonziliation der Unzuchtssünder, wie überhaupt der Kapitalsünder, kennt.

Wie mein verewigter Lehrer, so habe ich mich ebenfalls in meinen Studien wiederholt mit der altchristlichẹn Bußdisziplin befalst und in vorliegenden Falle seine Anschauung durch die Schrift Tertullians De baptismo bestätigt gefunden, wie er selbst in der Theol. Qu.-Schr. 1906,

I Die Bufschriften Tertullians De paenitentia und De pudicitia und das Indulgenzedikt des Papstes Kallistus, ein Beitrag zur Geschichte der Bußdisziplin 1905 (Bonner Programm).

2 Das Indulgenzedikt des Papstes Kallistus, Tübg. Theol. Quartalschrift 1906, $541-568$. 
554f. zustimmend crwähnt. Auch der französische Kirchenhistoriker Batiffol, der in scinen lítudes d'Histoire et de Théologic positıve, III édition 1904, 45-222 cine vorzügliche Darstellung der "Origines de la pénitence" gibt, stimmt im Bulletin de littérature ecclésiastique, publié par l'Institut catholique de Toulouse 1906, 339-48 Funks Ausführungen gegen Esser zu. Dagegen ist der Innsbrucker Dogmatiker Stufler S. J. vollauf überzeugt, dalb den Kapitalsündern von jeher in der Kirche einmalige Bußc mit Wiederaufnahme offengestanden sei (Ztschr. f. kath. Theol. 1906, 322 und 324 1907, 228 A. I; 372 ff, 433 ff, 577 ff; 1908, Iff), und Esser hält (Katholik 1907, II, 184 ff $297 \mathrm{ff}$ ) seine Anschauung gegen Batiffol und Funk aufrecht.

Auf protestantischer Seite ist man sich über diese Frage ebensowenig klar und einig geworden als auf katholischer. So fabt, um nur dịe neuesten Stimmen hier anzuführen, Harnack das Vorgehen der römischen Gemeinde als Neuerung, die dann von anderen Gemeinden angenommen worden sei, während Loofs ${ }^{2}$ in Tertullians Schrift De paenitentia die Wiederaufnahme der Büßer in die Kirche vorausgesetzt, und demgemäß die Indulgenz Kallists der bereits herrschenden Anschauung oder der bereits bestehenden Praxis durchaus entsprechend findet. Auf Stuflers emsige Bemühungen, den Kirchenhistorikern mit dogmatischem Rotstift die Hefte zu korrigieren, und auf seine eigentümlichen patristischen Beweisführungen gedenke ich andern Orts näher einzugehen. Hier möchte ich nur einen Punkt herausgreifen. In der Zeitschr. f. kath. Theol. 1907,614 schreibt er: „Auffallend ist es allerdings, daß in dritten Jahrhundert zwei Schismen sich gebildet haben, die zur Bußdisziplin der katholischen Kirche eine oppositionelle Stellung einnehmen: das Schisma des Hippolyt gegen Kallistus und das des Novatian gegen Kornelius. -Aber es muß mit Recht Befremden erregen, wenn katholische Historiker daraus den Schluß ziehen, daß jedesmal die Kirche die Neuerung eingefuhrt, das Schisma dagegen die alte Lehre beibehalten habe. Soll denn die ursprüngliche kirchliche Lehre gerade immer bei den Häretikern und Schismatikern zu suchen sein?" Und S. 617 läbt er den von Tertullian in seinem Beweisgang in De pudicitia so stark

I Die Mission und Ausbreitung des Christentums 2. A. 1906. I, 184.

2 Leitfaden zum Studium der Dogmengeschichte 4. A. 1906 S. 207. Es handelt sich um De paenit. c. 10: An melius est damnatum latere quam palam absolvi? Vgl. Funks Bemerkung zu dieser Stelle, Th. Qu.-Schr. 1906, 550f. Rolf fs (Das Indulgenzedikt des römischen Bischofs Kallist 1893, 40) findet in De paenit. c. 8 eine Wiederaufnahme, aber als Ausnahme. Preus chen schließt (Tertullians Schriften De paenitentia und De pudicitia mit Rücksicht auf die Bußdisziplin 1890, 14) mit einem non liquet. 
betonten Vorwurf, Kallist habe aus dem bisherigen Dreibund unvergebbarer Kapitalsünden unkonsequent allein die Unzucht herausgebrochen, einfach auf Unwahrheit beruhen. Einer solchen Methode gegenüber wird es gut sein, einmal einen Mann von unbestrittener Orthodoxie und Kirchlichkeit zu verhören, den Bischof von Lyon. Bei den Erörterungen über die altkirchliche Bußdisziplin wurden, zwar einige Stellen aus Irenäus bereits berïcksichtigt, aber nur gelegentlich, olne daß man seine Stellung zur Bußfrage im Zusammenhang seiner Gedanken ins Auge gefaßt hätte. Nun sagt der Bischof von Lyon über Verstoßung oder Wiederaufnahme schwerer Sünder direkt freilich gar nichts und auch über Buße und Sündenvergebung im allgemeinen nur wenig. Aber dieses Wenige läßt immerhin seine prinzipielle Anschauung durchblicken.

Man hat zum Beweise dafür, dab Irenäus eine Buße mit kirchlicher Rekonziliation kenne, auf einige Stellen verwiesen, wo er von der Bußleistung verschiedener von Häretikern verführter Frauen redet. ${ }^{x}$ So erzählt er Adv. haer. I, 6,3 (ed. Stieren I, 74) von den Valentinianern:

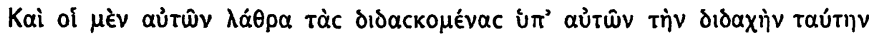

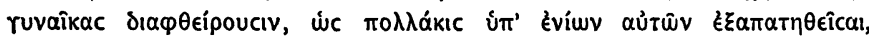

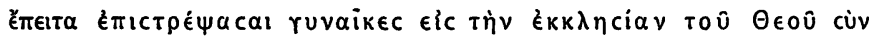

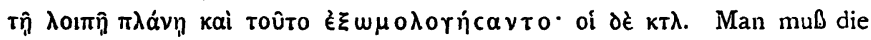
Möglichkeit, dal diese Frauen wieder ganz in die Kirche aufgenommen worden seien, zugeben. Abfall zu Häresie oder Schisma erscheint nirgends in der Reihe der dauernden Ausschluß begründenden Kapitalsünden, wie denn auch der Verfasser des kleinen Labyrinthes oder der Streitschrift gegen Artemon (bei Eusebius H. E. V, 28) von der Bulje und Wiederaufnahme des Konfessors Natalis, der sich zum Bischof der Monarchianer hatte weihen lassen, berichtet." Bei den von den Gno-

I So schon der Franziskaner Feuardent in seinen Anmerkungen zu Irenäus (bei Stieren II, $646 \mathrm{sq}$ ), ferner der Oralorianer Morin in seinem $165 \mathrm{l}$ erschienenen Commentarius historicus de disciplina in administratione sacramenti poenitentiae II, 10, 8 (Venediger Ausgabe von 1702 S. 66), und der Mauriner MI assuet in seiner Dissertatio III de Irenaei doctrina, art. VII Nr. 74 und 75 (bei Stieren II, 306 sqq), neuestens Loofs, J.eitfaden zum Studiuan der Dogmengesch. 4 A. 1906 S. 206.

$=$ Vgl. Funk, Kirchengesch. Abh. u. Unters. I, 166f. Wie nachsichtig man gegen reuige Häretiker sein konnte, zeigt der Bericht des Irenäus über den Gnostiker Cerdo,

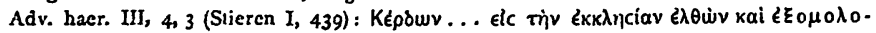

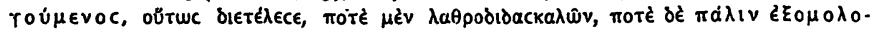

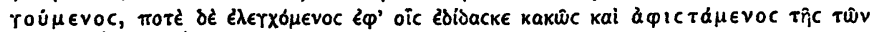
dofiqūv cuvodiac. Es ist freilich eine irrige Annahme, dals er nach dem ersten Bufbekenntnis gleich in die kirchliche Gerneinschaft aufgenommen worden sei, von Stuflers Deutung, daf die Aufnahme nach crneutem Abfall wiederholt worden sei (Zeitschr. $f$. 
stikern verführten Frauen kamen allerdings zu den dogmatischen noch sittliclie Verirrungen. Aber diese standen mit jenen in innigstem $\mathrm{Zu}$ sammenlıang, waren die Betätigung libertinistischer, aus dem ganzen

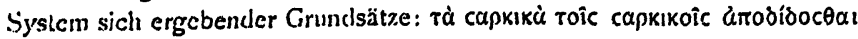

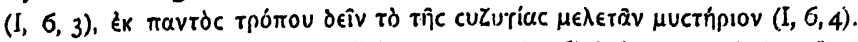
Indes redet Irenäus ausdrücklich nur von der Bckehrung und dem Bekenntnis, nicht von der vollen Wicderaufnahme der gefallenen Frauen.

So heißt es auch I, 13,5 (Stieren I, 152) von den durch den Gnostiker

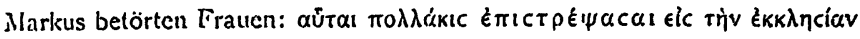

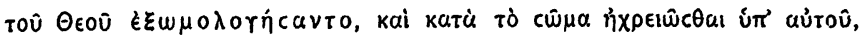

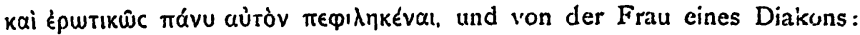

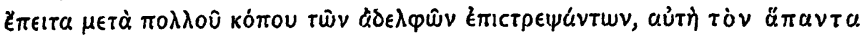

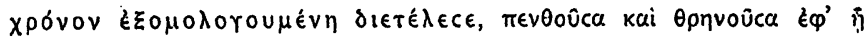

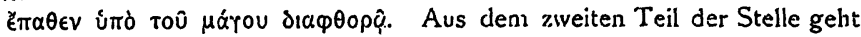
hervor, daß Ł६opoloreic $\theta a$ r bei Irenäus, wie exomologesis bei Tertullian De paenit. c. 9, nicht allein das Bekenntnis, sondern die ganze Bufleistung bezeichnet. Wie lange die Buße der Diakonsfrau währte, ist nicht recht klar. Denn tòv ü $\pi \alpha v \tau \alpha$ xpóvov kann bedeuten "die ganze ihr festgesetzte Zeit", oder "die ganze Zeit" = lebenslänglich. Und hat auch die zweite Deutung die größere Wahrscheinlichkeit für sich, so bleibt sofort wieder dahingestellt, ob diese lebenslängliche Bufe der Frau von der Kirche diktiert wurde ohne Aussicht auf kirchliche Rekonziliation (oder höchstens unmittelbar vor dem Tode), oder ob sie die Buße auch nach erfolgter Rekonziliation freiwillig noch fortsetzte. Alle diese Möglichkeiten bleiben an sich offen. IVurden die Frauen wieder in die Kirchengemeinschaft aufgenommen, so konnte als strafmildernd nicht bloß der Zusammenhang ihrer Fehltritte mit der gnostischen Lehre, sondern auch der Umstand angesehen werden, dafs sie eben den Liebestränken und Zauberkünsten des Magiers erlegen waren. ${ }^{\text {s }}$

kath. Th. 1907, 440 f), ganz zu schweigen. Die Kirche war kein Taubenschlag, wo man nach Belieben aus und ein fliegen konnte. Die Worte des Irenäus verraten vielmehr ziemlich deutlich, daß Cerdo über die Exomologese nie hinauskam, sondern noch vor der völligen Aufnahme wieder seine I,ehre verbreitete, um dann wieder zur Buße zu kommen und schließlich, der Irrlehre überfülurt, ganz abzufallen. Aber immerhin hatte er die durch Rückfall unterbrochene Bufe fortsetzen dürfen.

I Es ist wiederholt, so auch ron Gartmeier (Die Beichtpflicht 1905 S. 8I), der Finger darauf gelegt worden, daß diese Frauen selbst die verborgensten Umstände, wie ihre leidenschaftliche Liebe zu Markus, geoffenbart hätten. Allein Irenäus fügt diesen Umstand lediglich zum Beweis der unwiderstehlichen Wirksamkeit der Zaubermittel des

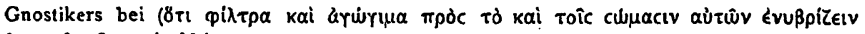
$\epsilon u \pi 01 \epsilon \hat{\imath}$ oũroc $\delta$ Mápкoc). Die Frauen in I, 6, 3 sündigten lediglich aus gnostischem 
Endlich heift es I, I3,7 (Stieren I, I58) von den weiblichen Opfern

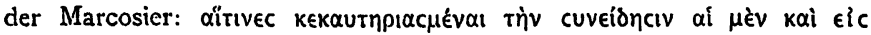

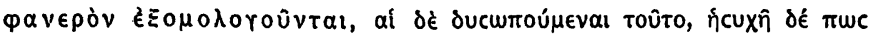

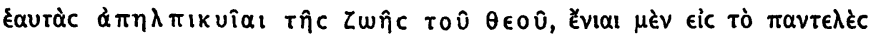

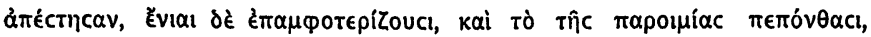

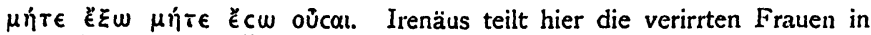
zwei Klassen: solche, die öffentliche Buße leisten und solche, die sich davor schämen und verzweifeln. Von diesen letzteren fallen die einen ganz vom Christentum $a b$, während die andern schivanken und so nach dem Sprichwort "weder draus noch drin sind“. Das eccu nötigt keineswegs, wie Loofs glaubt, zur Annahme einer kirchlichen Rekonziliation, sondern kann sich auch, wie Funk (Theol. Qu.CChr. 1906, 551 A. 1) bemerkt, auf die Bußleistung beziehen. Eine sprichwörtliche Redensart darf in der Anwendung nicht gepreßt werden, ${ }^{x}$ wenn man von ihr auch größere Sinngemäßheit verlangen kann als von den Spruchhäufungen Sancho Pansas.

Außer diesen Stellen wurde noch auf adv. haer. IV, 40, I verwiesen:

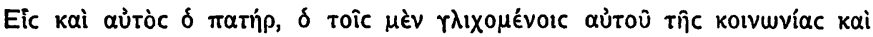

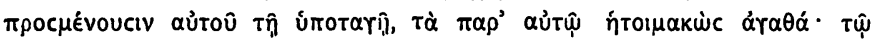

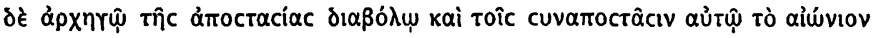

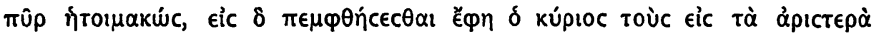

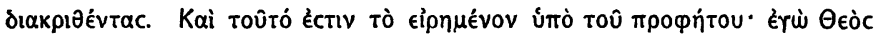

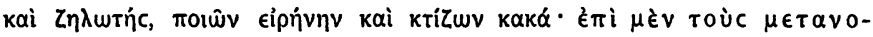

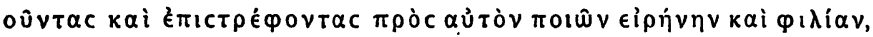

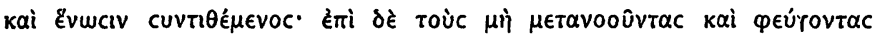

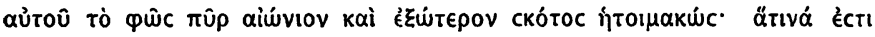

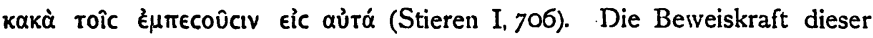
Stelle für eine kirchliche Rekonziliation der Kapitalsünder hat schon Funk abgewiesen, da sie zu allgenein laute und einfach mit Bezugnahme auf Jes. 45,6f. von der Bekehrung zu Gott und dem so gewonnenen Frieden rede (Abh. u. Unters. I, I67). Irenäus verteidigt gegenüber dem Dualismus der Gnostiker den christlichen Monismus: derselbe Gott teilt Lohn und Strafe aus, Lohn den Guten, Strafe den Bösen. Das ist der einfache Sinn. Schwebt ihm je ein kirchliches Gnadenmittel vor, so

Prinzip, wie Frau von Warens in Rousseaus Bekenntnissen, die andern I, 13, 5 aber auch in leidenschaftlicher, ihnen angezauberter liebe. Irenäus zweifelt an dein Zauber keinen Augenblick. So energisch er den Gnostizismus bekämpft - diesem dunkeln Erbstick des Hellenismus steht sein Geist doch offen.

I Massuet bemerkt zur Stelle einfach: Proverbium de iis dictum, qui ancipitis ac incerti animi hresitant, quid potissimum eligant (bei Stieren I, 158 note). 
ist es nicht die Buße, sondern die Taufc. Die Menschen, die „Bubie tun und sich zu Gott bekehren", sind mit denen, die "nach seiner Gcmeinschart strcben und im Gehorsam verharren", identisch, wie "die nicht Bubc Tuenden und sein Licht Fliehenden" und "die zur Linken Gestelltcn", die zum Fürsten des Abfalls und zu den mit ihm abgefallenen Engeln ins ewige Feuer wandern, dieselben sind. Das primäre Sakranicnt der Buble und Bekehrung und der Sündennachlassung ist, wie aus der heiligen Sclurift, dem Hirten des Hermas, Tertullian, Cyprian klar hervorgeht, die Taufe. Von diesem Gesichtspunkte aus könnte man in dieser Stelle sogar das Gegenteil von einer kirchlichen Rekonziliation finden, da nur den im Gehorsam Beharrenden (und demnach die Taufgnade Bewahrenden) das Heil zugesprochen erscheint. Doch sei darauf keinerlei Gewicht gelegt. Es genügt die Konstatierung, daß aus der Stelle keine kirchliche Wiederaufnahme der Kapitalsünder erschlossen werden kann.

Dasselbe gilt von der großenteils gleichlautenden Stelle III, 23, 3 (Stieren I, 548): Hoc idem autem et Dominus in Evangelio his, qui a sinistris inveniuntur, ait: Abite maledicti in ignem aeternum, quem praeparavit Pater meus diabolo et angelis ejus; significans, quoniam non homini principaliter praeparatus est aeternus ignis, sed ei qui seduxit et offendere fecit hominem, et, inquam, qui princeps apostasiae est, principi abscessionis, et his angelis qui apostatae facti sunt cum eo; quem quidem juste percipient etiam hi qui, similiter ut illi, sine poenitentia et

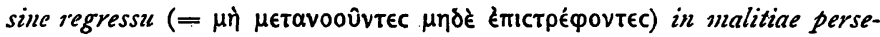
verant operibus.

Es fallt einigermaßen auf, mit welchem Nachdruck Irenäus betont, daß nur Gott Sünden nachlasse. Adv. haer. V, 17, I setzt er auseinander, daß derselbe Gott, gegen den die Menschen durch Gesetzesübertretung gefehlt hätten, durch Vermittlung des Sohnes gnädig gestimmt, die gegen ihn begangenen Sünden verziehen habe. Quomodo autem vere remissa sunt peccata, nisi ille ipse, in quem peccavimus, donavit remissionem, per viscera misericordiae Dei nostri; in quibus visitavit nos per Filium suum? (Stieren I, 763). Und nachdem von Matth. 9, 6 die Rede war, heißt es vom Heiland V, 17, 3: Peccata igitur remittens, hominem quidem curavit, semetipsum autem manifeste ostendit quis' esset. Si enim ncmo potest remittere peccata, nisi solus Deus, remittebat autem haec Dominus et curabat homines: manifestum, quoniam ipse erat verbum Dei, Filius hominis factus, a Patre potestatem remissionis peccatorum accipiens, quoniam homo et quoniam Deus: ut quomodo 
homo compassus est nobis, tanquam Deus misereatur nostri et remittat nobis debita nostra, quae factori nostro debemus Deo (Stieren I, 769). Solch exklusive Sprache ist einer kirchlichen Sündenvergebung und Wiederaufnahme nicht gerade günstig, wenn auch anderseits zu beachten ist, daß Irenäus in seinem dortigen Beweisgang keine Veranlassung hatte, auf die kirchliche Lösegewalt Bezug zu nehmen. Soviel ist sicher, dal' der Gedankengang "der Stelle auf keine wiederholte Sündennachlassung hinausläuft, sondern auf die durch den Erlösungstod Christi einmal objektiv vollzogene Sündentilgung, die David (Ps. 3I, If) vorausgesetzt habe: eam, quae per adventum ejus est, remissionem praemonstrans, per quam delevit chirographum debiti nostri, et affixit illud cruci, uti quemadmodum per lignum facti sumus debitores Deo, per lignum accipiamus nostri debiti remissionem.

Damit kommen wir auf eine Stelle, die uns die Gedanken des Kirchenvaters deutlich verrät, und von der man sich wundern mul., dafis sie für unsere Frage nirgends angezogen wurde, Adv. haer. IV, 27 (Stieren I, 648ff). Nachdem er vorher die Fehltritte der Patriarchen und Propheten berührt hat, fuihrt er aus: dem Worte eines Presbyters zufolge dürfen wir diese Alten darob nicht tadeln, es genügt vielmehr ihre Zurechtweisung in der Schrift. Als David gesündigt hatte, kam der Prophet Nathan, hielt ihm seine Sünde vor und bewog ihn zur Reue und Buße. Darauf dichtete David den Bußpsalm (psalmum exhomologeseos) in Erwartung der Ankunft des Herrn, qui abluit et emundat eum hominem qui peccato fuerat abstrictus. Auch Salomo wird wegen seiner Liebe zu fremdländischen Weibern von der Schrift hinlänglich ausgescholten, damit sich kein Fleisch vor dem Herrn rühme (IV, 27, 1).

Darum ist der Herr in die Unterwelt hinabgestiegen, um auch dort seine Ankunft $z u$ verkünden, remissione peccatorum exsistente his qui credunt in eum. Es glaubten an ihn aber alle, die auf ihn hofften, die seine Ankunft vorherverkündigten und seinen Anordnungen dienten, die Gerechten und Propheten und Patriarchen. Ihnen hat er geradeso wie uns die Sünden nachgelassen, und wir dürfen ihnen also, wenn anders wir die Gnade Gottes nicht verachten wollen, ihre Sünden nicht anrechnen. Denn wie jene uns unsere Verfehlungen nicht anrechneten, die wir vor Christi Erscheinung begingen, so wäre es auch von uns ungerecht, denen, die vor der Ankunft Christi sündigten, das anzurechnen. ${ }^{x}$

"Nos" hat bei Irenāus bald eine weitere, bald eine engere Bedeutung. Hier sind damit, wie Grabe (bei Stieren I, 65I not. 3) richtig angibt, die Heiden vor und naçh der Ankunft Christi oder vor und nach ihrer Bekehrung gemeint. „Priusquam 
Denn allc entbchren des Ruhmes vor Gott (Röm. 3, 23); es werden aber. niclit atus sich sclbst, s'sndern durch die Ankunft des Herrn, gerechtfertigt die nach scinem Liehte streben. Der Altväter Taten aber sind zu unserer Warnung aufgezeichnct, fürs erste, damit wir wülten, dal unser und ihr Gott cin und derselbe ist, dem Sünden auch hervorragender Menschen mibfallen, sodann, danit wir uns vor allem Bösen hüteten. Si cnim hi qui praccesserunt nos in charismatibus veteres, propter quos nondum Filius Dei passus erat, delinquentes in aliquo, et concupiscentiae carnis servientes tali affecti sunt ignominia; quid passuri sunt qui nunc sunt, qui contemserunt adventum Dumini et deservicrunt voluptatibus suis? Et illis quidem curatio et remissio peccatorum mors Domini fuit; propter cos vero, qui mens peccant, Christus non jam 11norietur, jam enim mors non dominabitur ejus; sed veniet Filins in gloria Patris, exquirens ab actoribus et dispensatoribus suis pecuniam, quam eis credidit, cum usuris, et quibus plurimum dedit, plurimum ab eis exiget. Non debemus ergo, inquit ille senior, superbi esse, neque reprehendere veteres; sed ipsi timere, ne forte post agnitionem Christi agcntes aliquid, quod non placeat Deo, remissionem ultra non habeamus delictorum, sed cxcludamur a regno ejus. Et ideo Paulum dixisse (Röm. I I, 21. 17): ,Si enim naturalibus ramis non pepercit, ne forte nec tibi parcat, qui quum esses oleaster, insertus es in pinguedinem olivae, et socius factus es pinguedinis ejus" (IV, 27,2).

Also die Alten haben durch den Tod Christi Verzeilhung der Sünden erlangt. Wer aber jetzt, nachdem er durch Jesu Tod Sündennachlassung erlangt und die Erlösung an sich erfahren hat, sündigt, hat von Jesu Tod nichts mehr zu hoffen, da Jesus nicht nochmals für ihn sterben kann, sondern nur sein Gericht $z u$ fürchten. Wir sollen darum jene Alten nicht tadeln, vielmehr für uns selber fürchten, wir könnten, nachdem wir Christus erkannt haben, in eine Sünde fallen und dann keine Sindennachlassung mehr erlangen, sondern vom Reiche Gottes ausgeschlossen werden. So Irenäus im Anschluß an seinen "Presbyter“. "

Christus in nobis manifestaretur" bezeichnet 2 war zunächst die Erscheinung des Herrn auf Eden (rgl. III, 18, 7: qui dicunt eum putative manifestatum, der gnostische Doketismus), schliebt aber auch die Offenbarung Christi in der Heidenwelt $\mathrm{d}$. h. ihre Bekehrung ein.

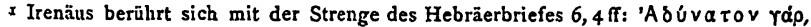

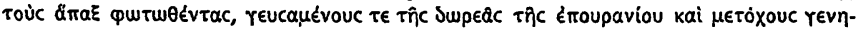

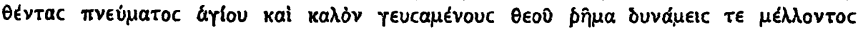

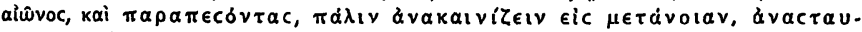

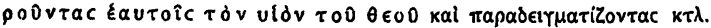


Hier ist nach dem klaren Wortlaut für eine nach der Taufe begangene (Kapital)sünde die göttliche Verzeihung wenn nicht ausgeschlossen, so doch stark in Frage gestellt. Von einer kirchlichen Rekonziliation kann bei solcher Anschauung keine Rede sein. Denn die durch den protestantischen Theologen Steitz (Das römische Bußsakrament $\mathrm{I} 854,48 \mathrm{ff}$ ) und den altkatholischen Theologen Götz (Die Buflehre Cyprians I \$95, $" 44$ ff) vertretene Auffassung, daß die Rekonziliation im Altertum nur dic IViedererlangung der kirchlichen Gemeinschaft bedeute und zur göttlichen Verzeihung in keiner Beziehung stehe, stellt, wie auch Karl Müller (Ztschr. f. KG. I6 (1896) I87ff. Theol. Litztg. 1897, $463 \mathrm{ff})$ betont, den geschichtlichen Tatbestand geradezu auf den Kopf. Die kirchliche Rekonziliation setzt die göttliche Verzeihung voraus und schliebt sie ein, ist Erklärung und Zuwendung der göttlichen Sündennachlassung. Die älteste Kirche versagte ihre Gemeinschaft, selbst wo sie dem Sünder bei lebenslänglicher Buße göttliche Verzeihung in Aussicht stellte. Wo, wie hier bei Irenäus, sogar die Gnade Gottes verneint oder wenigstens stark in Frage gestellt wird, kann von einer Wiederaufnahme in die kirchliche Gemeinschaft vollends keine Rede sein.

Wie ernst es unserm Kirchenvater ist, und welche Sünden er im Auge hat, zeigt der Fortgang seiner Erörterung. IV, 27, 3: Similiter et plebis praevaricationes vides descriptas esse, non propter illos, qui tunc transgrediebantur, sed in correptionem nostram, et ut sciremus unum et eundem Deum, in quem illi delinquebant, et in quen nunc delinquint quidam ex his qui credidissc dicuntir (folgt I Cor. IO, Iff). IV, 27, 4: Quemadmodum ibi in pluribus eorum, qui peccaverunt, non bene sensit Deus, sic et hic ,vocati multi, pauci vero electi": quemadmodum ibi injusti et idololatrae et fornicatores vitan perdidernut, sic et hic, et Domino quidem praedicante, in ignem aeternum mitti tales, et apostolo dicente (I Cor. 6,9f). Et quoniam non ad eos, qui extra sunt, hoc dicit, sed ad nos, ne projiciamur extra regnum Dei, tale aliquid operantes, intulit (I Cor. 6, II). Et quemadmodum illic condemnabantur et projicicbaintur hi, qui male operabantur et reliquos exterminabant, similiter et hic oculus quoque effoditur scandalizans et pes et manus, ne reliquum corpus pariter pereat (Matth. I8. 8f; es folgen I Cor. 5, II. Ephes. 5, $6 \mathrm{f}$. I Cor. 5, 6. Röm. I, I8. Luk. I8, 7 f. II Thess. I, 6ff). IV, 28, I: Quum ergo hic et illic eadem sit in vindicando Deo justitia Dei; et illic quidem typice et temporaliter et mediocrius, hic vero verc et semper et austerius; ignis enim aeternus et quae a caelo revelabitur ira Dei a facie Domini nostri ... majorem poenam praestat is qui incidunt in eam. 
IV, 28, 2: Sic et poena eorum, qui non credunt Verbo Dei ct contemnunt cjus adventum et convertuntur retrorsum, adampliata est, non solum temporalis, sed ct actcrna facta .... adjudicans eos qui adjudicantur, id est cos, qui obliviscuntur Deum et sunt blasphemi et trans. gressoris ejus verbi.

Das sind Sätze, die einen dauernden Ausschluf der Kapitalsünder aus der Kirchengemeinschaft erkennen lassen. In ihrem Lichte erhält auch die oben erwähnte Exklusivität, womit Irenäus die göttliche Sündennachlassung hervorhebt, ihren vollen Sinn: die Kirche hält die Sünder zwar zur Bube an, enthält sich aber eines Urteils über sie und stellt dieses Gott anheim. Irenäus zweifelt stark an ihrem Heil.

In dieselbc kirchliche Umwelt, dieselbe Höhenluft gesteigerter Sittlichkeit und innerer Reinheit versetzt uns die begeisterte Schilderung des Irenäus in seiner neuentdeckten Schrift „Zum Erweise der apostolischen Verkündigung“ c. 95 u. 96: „Wir haben den Herrn des Gesetzes, den Sohn Gottes, empfangen. Und durch den Glauben ạn ihn lernen wir Gott von ganzem Herzen lieben und den Nächsten wie uns selbst. Die Liebe zu Gott aber ist ferne von aller Sünde und die Liebe zum Nächsten fügt dem Nächsten kein Übel zu. Darum haben wir auch kein Gesetz zum Erzieher nötig; siche, wir sprechen mit dem Vater und stehen ihm von Angesicht zu Angesicht gegenüber, Kinder geworden an Bosheit und erstarkt in Gerechtigkeit und Wohlanständigkeit. Denn das Gesetz wird nicht mehrsagen: ,Du sollst nicht ehebrechen dem, dem nicht einmal mehr eine Lüsternheit zu dem Weibe des Fremden (in den $\operatorname{Sin} n$ ) kommt; und nicht, Du sollst nicht töten' dem, der jeden Zorn und jede Feindseligkeit von sich entfernt hat; und nicht ,Du sollst nicht Verlangen tragen nach deines Ṇächsten Hof noch nach seinem Ochsen oder seinem Esel' denen, die sich um die irdischen Dinge gar nicht kümmern, sondern himmlische Früchte sammeln; und nicht ,Auge um Auge, und Zahn um Zahn' demjenigen, der niemanden für seinen Feind, sondern alle für seine Nächsten hält; deshalb kann er nicht einmal seine Hand zur Rache ausstrecken."

Der Bischof von Lyon ist ein Mann von anerkannter Rechtgläubigkeit und strenger Kirchlichkeit. Aus seinen Worten spricht noch einmal - kurz bevor es erstmals offiziell herabgestimmt wurde - das Hochgefühl einer Kirche, die eine Gemeinșchaft von Heiligen bilden will und mit

In armenischer Version entdeckt, herausgegeben und ins Deutsche übersetzt von Karapet Ter-Mlekerttschian und Erwand Ter-Minassiantz, Texte u. Untersuch. XXXI, I. 1907 , S. 49 f. 
diesem Grundsatze vollen Ernst macht, die darum keinen Unreinen in ihrer Mitte duldet und keinen schwer Gefallenen wieder ganz aufnimmt. Mit der Wende des zweiten Jahrhunders erlahmte die Schwungkraft, verblaßte das Ideal, und die menschliche Schwäche der in die Kirche einströmenden Menge machte sich geltend. Bald verkündete Kallist sein "Ego et moechiae et fornicationis delicta poenitentia functis dimitto“ (Tert. de pudic. I), und half damit der Kirche über eine Krisis hinüber. Das zähe Festhalten am urchristlichen Ideal wurde jetzt eine Marotte von Schwärmern und Sektierern, die den Wandel der Zeiten und das gute Recht geschichtlicher Entwicklung verkannten, der Montanisten, Novatianer, Donatisten. Die Kirche aber gewann nit der Reduzienung ihrer sittlichen Anforderungen und mit der Zunahme ihrer Nachsicht gegen die menschliche Schwäche an Popularität und Anziehungskraft. Sie wurde Weltkirche und wollte an den weltflüchtigen Enthusiasmus der Anfänge, ihren eigenen goldenen Jugendtraum, fortan nicht mehr erinnert werden. Es stand nicht lange an, bis man bei der bekannten Neigung, die eigene Praxis in die apostolische Zeit zurückzuveriegen, mit überraschender Zuversicht behauptete, die einmalige Wiederaufnahme schwerer Sünder sei in dor Kirche immer üblich gewesen. $\mathrm{Da}$ sich aber gegen das Edikt des Papstes Kallist Opposition erhob, und namentlich ein heißblütiger Eiferer wie Tertullian die schärfsten Töne dagegen anschlug, darf nicht Wunder nehmen. Es bedeutete eben eine Milderung und Neuerung, einen Abstrich vom urchristlichen Ideal. Vor Tisch las man anders - wie in Karthago, so auch in Lyon, wo der strenge Kirchenmann Irenäus den Hirtenstab führte.

Noch eine Bemerkung über die Tragweite der irenäischen Anschauung sei hier beigefügt. Vor kurzem hat P. A. Kirsch in einem Aufsatz über „Die Behandlung der crimina capitalia in der morgenländischen Kirche im Unterschied zur abendländischen" (Archiv f. kath. Kirchenrecht 84 [1904] 263-282) den Nachweis zu führen gesucht, daß die orientalische Kirche, im Unterschied von der abendländischen, von Anfang an sämtlichen Kapitalsündern einmalige Buße und Wiederaufnahme gewährt habe. ${ }^{2}$

So beachtenswert seine Argumentation ist, so bleiben doch schwere

I Ich wundere mich, dab Funk weder in der neuesten Auflage seiner Kirchengeschichte 1907, 59, noch sonst irgendwo Notiz davon genommen hat. Die Abhandlung scheint ihm ebenso entgangen $2 u$ sein, wie seinem Gegner Stufler, der sie in seinem Aufsatz über „Die Sündenvergebung bei Origenes" (Ztschr. f. kath. Theol. 1907, 193-228) nicht erwähnt, obwohl Kirsch auch die Stellung des Alexandriners zur Bubfrage berücksichtigt. 
Bedenken übrig, von denen hier nur eines seinen Platz hat. Irenäus lat seine Anschauung von der Wirkung der Todsünden sicher nicht erst im Abendland gewonnen, sondern schon aus seiner Heimat Kleinasien mitgebracht. Wir könnten das behaupten, wenn cr es auch nicht ausdrücklich sagte. Er beruft sich aber tatsächlich auf „einen Presbyter der es von denen gehört hatte, die die Apostel noch gesehen und ven ilinen gelernt hatten" (adv. haer. IV, 27, I. Stieren I, 648), also auf seine kleinasiatische Schule. Daraus ergibt sich jedenfalls soviel, dab die Bußfrage in der ältesten orientalischen Kirche nicht die einheitliche Lösung fand, die Kirsch annimmt. Man wird also zum mindesten mit verschiedenen Strömungen und verschiedener Praxis rechnen müssen. 